Relations industrielles

Industrial Relations

\title{
Jurisprudence du travail
}

Volume 5, numéro 10, juillet 1950

URI : https://id.erudit.org/iderudit/1026385ar

DOI : https://doi.org/10.7202/1026385ar

Aller au sommaire du numéro

Éditeur(s)

Département des relations industrielles de l’Université Laval

ISSN

0034-379X (imprimé)

1703-8138 (numérique)

Découvrir la revue

Citer cet article

(1950). Jurisprudence du travail. Relations industrielles / Industrial Relations, 5(10), 97-98. https://doi.org/10.7202/1026385ar

Tous droits réservés @ Département des relations industrielles de l’Université Laval, 1950
Ce document est protégé par la loi sur le droit d'auteur. L'utilisation des services d'Érudit (y compris la reproduction) est assujettie à sa politique d'utilisation que vous pouvez consulter en ligne.

https://apropos.erudit.org/fr/usagers/politique-dutilisation/ 


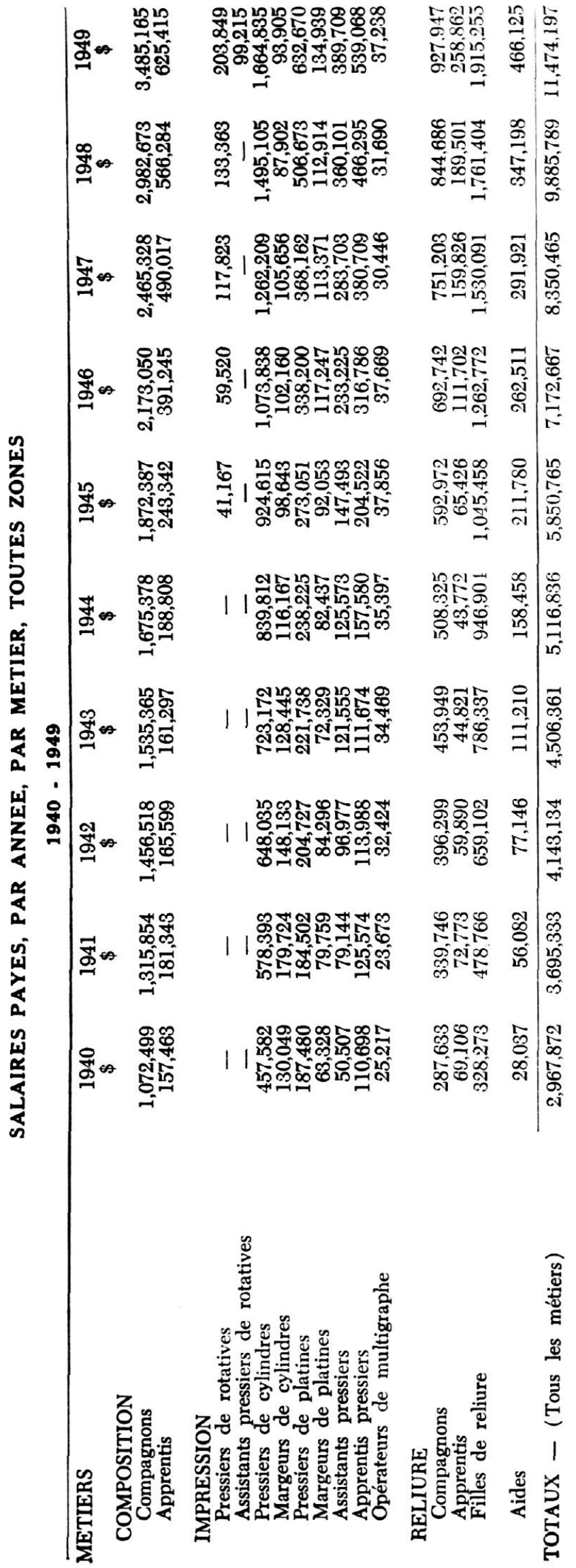

\section{JURISPRUDENCE DU TRAVAIL}

\section{Prescription et lois ouvrières}

\&e 17 août 1945, la demanderesse s'engagea comme institutrice pour les défendeurs, à raison de $\$ 400.00$ par année. Elle exécuta son contrat et reçut la somme de $\$ 400.00$. La Commission scolaire de St-Philippe est dans le district d'inspection no 60 . Les institutrices de ce district formèrent une association suivant la Loi des relations ouvrières et la Loi des différends entre les services publics et leurs salariés (S.R.Q. 1941, Ch. 162A et 169). L'association est munie d'un certificat en date du 2 août 1944 et ainsi est agent de négociation des institutrices catholiques au service des défendeurs. Les parties n'ayant pu négocier un projet de convention collective, le ministre ordonna la formation d'un conseil d'arbitrage afin de régler le différend. Le Conseil d'arbitrage rendit sa décision le 11 février 1946. La sentence arbitrale fut déposée entre les mains du ministre du travail et communiquée aux défendeurs. Elle constitue un titre en faveur de la demanderesse, tel que décidé par la Cour d'appel dans Association catholique des institutrices rurales du district no 60 vs Commissaires d'écoles de St-Pascal (1947 BR 728). La demanderesse, selon cette sentence, avait droit à la somme de $\$ 730.00$. Il lui est dû une solde de $\$ 330.00$ qu'elle réclame. $>$

Le débat est ramené à une question de prescription par la défense qui prétend, pour cette cause, que la sentence arbitrale est inopérante.

Or voici en substance le jugement. La Loi des relations ouvrières, la Loi des différends ouvriers de Québec et la Loi des différends entre les services publics et leurs salariés n'édictent aucune prescription de salaires. Par ailleurs, la Loi des syndicats professionnels, la Loi de la convention collective et la Loi du salaire minimum qui édictent une prescription de six mois, ne s'appliquent pas dans l'espèce. Il faut donc recourir à l'art. 2260, par. 6, C.C. Dès lors, la réclamation d'une institutrice pour le paiement de l'augmentation de son salaire fixée par une sentence arbitrale se prescrit par cinq ans, les parties admettant qu'il n'y a jamais eu de convention particulière.

(Mlle Gagnon vs Les commissaires d'écoles pour la municipalité de Saint-Philippe, Kamouraska, 24 janv. 1949; Président, Juge Langlais; avocat de la demanderesse, Me M.-L. Beaulieu, c.r.; avocat des défendeurs, Me V. Trépanier, c.r.; cf. R.J. de Q., C.S., 1949, p. 201.)

\section{Sécurité syndicale}

Dans un récent arbitrage, les membres du tribunal rejettent à l'unanimité une demande « d'atelier d'union * en invoquant les raisons suivantes: « La liberté individuelle pour laquelle les unions elles-mêmes se sont battues dans le passé, liberté individuelle qui est actuellement sanctionnée par le Traité des droits de d'homme, signé par notre pays et l'économie de nos lois, ne semble pas pouvoir nous autoriser à accorder cette demande $\$$.

(McLennan Lumber Limited, Montréal, et l'Association canadienne des travailleurs du bois, local no 16, Inc.; sentence unanime, le 18 juillet 1949; président, M. le juge Irénée Lagarde; arbitre patronal, Me Philippe Lamarre, c.r.; arbitre syndical, M. Léopold Lavoie.) 
Les rapports judiciaires de Québec publiés par le Barreau de la province résument ainsi un jugement rendu par le juge Pettigrew de la Cour des sessions de la paix:

La Loi des relations ouvrières est intravires des pouvoirs de la Législature provinciale. N'est pas incompatible avec l'art. 502-A du Code criminel et, par conséquent, n'est pas inopérant l'art. 44 de la Loi des relations ouvrières qui prescrit une pénalité pour le défaut de se conformer aux obligations que cette Loi impose. Les corporations comme les individus, peuvent commettre une infraction criminelle. Bien que les commissions scolaires puissent ne pas réengager les instituteurs sans être tenues de donner des raisons de leur décision, elles sont cependant soumises aux prescriptions de l'art. 21 de la Loi des relations ouvrières et ne peuvent refuser d'employer une institutrice parce que, comme dans l'espèce, elle est présidente d'une association de salariés. L'autorisation de la Commission de relations ouvrières, requise par l'art. 49 de la Loi des relations ouvrières, est suffisamment libellée s'il appert que la Commission a donné sa décision en toute connaissance de cause. Dans l'espèce, elle renferme implicitement le texte de la requête et, conséquemment, contient tous les

\section{LES LIVRES}

El Contrato Colectivo de Trabajo, su significado economico-social, Mexico 1949; Modernization de las Relationes de Trabajo, aspectos de una nueva conciencia patronal, Mexico 1949. Ces deux ouvrages d'environ 60 pages chacun contiennent le compte rendu des Congrès de la Confederacion Patronal de la Republica Mexicana. Le premier étudie la convention collective de travail que l'on considère en général, par rapport aux parties contractantes, à l'entreprise et à l'économie nationale. Le second a trait à la modernisation des relations du travail et étudie surtout le rôle du patron dans ses relations avec les travailleurs, comme chef d'entreprise et par rapport à l'économie nationale. Les expériences par lesquelles les patrons mexi-

\section{STATISTIQUES ET INFORMATION}

\section{JOURS FERIES PAYES DANS LES CONVENTIONS}

Voici une troisième et dernière tranche du travail fait en collaboration avec l'office de recherches du Département des relations industrielles de la Faculté des sciences sociales de l'Université Laval et portant sur les jours fériés payés.

Le premier tableau nous donne une vue d'ensemble des clauses de jours fériés payés incluses dans 463 conventions coilectives de travail déposées à la Commission de relations ouvrières de la province de Québec. Ces conventions couvrent tous les groupes d'établissements sauf les industries manufacturières, réparties selon le nombre d'employeurs accordant ces congés payés et d'employés intéressés. Toutes ces conventions étaient en vigueur au 31 décembre 1948.

Nous y constatons que 944 employeurs sur 1,930 ayant à leur emploi 28,806 travailleurs sur un total de 59,111 accordent de deux à quinze jours fériés payés par année. Par contre, 986 de ces 1,930 employeurs occupant 30,305 des 59,111 travailleurs n'accordent aucun congé payé. Il est facile aussi de constater que les fêtes religieuses dominent. éléments essentiels. Sous la Loi des convictions sommaires de Québec, la motion de cassation de la plainte est recevable seulement lorsqu'elle soulève des irrégularités qui ne peuvent être corrigées par amendement.

(Mlle Couture v. Commissaires d'école pour la Municipalité scolaire de Lauzon; M. le juge Pettigrew; Cour des sessions de la paix, no 37,801 , Québec, le 30 mars 1950, R. B. de Q., C.S., maijuin 1950, p. 201 sq.)

\section{Vacances payées, facteur de stabilité}

Dans un arbitrage qui a eu lieu en juillet 1949, les arbitres accordaient à l'unanimité une seconde semaine de vacances payées après cinq ans de services continus. Les arbitres donnaient pour raison qu'ils avaient en vue d'assurer une plus grande stabilité chez les employés de la Compagnie.

(Radiateur Plessis Limitée et le syndicat des employés de fonderies de Plessisville, Inc.; président, Me Roger Thibaudeau; arbitre patronal, Me Maurice Boulanger, C.A.; arbitre syndical, Me Marius Bergeron; sentence unanime, 22 juillet 1949.)

cains sont passés il n'y a pas si longtemps lorsqu'ils se sont vus en face d'un gouvernement à la merci de syndicats révolutionnaires, et la paix relative dans laquelle ils se trouvent actuellement donnent à ces travaux un caractère de réalisme et un sens des responsabilités sociales chrétiennes que l'on souhaiterait voir prendre aux groupements patronaux de notre pays.

Cette confédération patronale qui groupe la très grande majorité des employeurs mexicains ne croit pas que pour réaliser la paix dans les relations du travail, même en face de syndicats qui sont loin de s'inspirer de la doctrine de l'Eglise, il faille créer une atmosphère de névrose et organiser un patronat de combat.

G.D.

\section{COLLECTIVES DE LA PROVINCE DE QUEBEC}

Les règles d'application suivantes se retrouvent dans quelques conventions. Ainsi quatorze de ces conventions exigent la présence au travail la veille et le lendemain du congé pour pouvoir bénéficier de la rémunération. Une convention exige la présence au travail pendant toute la semaine précédant le congé. Le minimum de service requis pour avoir droit aux fêtes payées stipulées dans la convention est de un mois dans une convention, de trois mois dans trois conventions, et d'un an dans quatre.

Quatre conventions n'accordent que demi-rémunération pour chaque jour de fête chômée. Une seule convention prévoit la compensation par un congé additionnel pour le jour férié survenant pendant la vacance annuelle d'un travailleur. Cent cinquante-trois conventions collectives déterminent l'observance des fêtes et stipulent une règle spéciale de rémunération pour le travail effectué ce jour-là.

Le deuxième tableau nous donne l'état des congés payés dans 702 conventions collectives de travail dans les industries manufacturières de la province de Québec. Ces conventions étaient en vigueur au 31 décembre 1948. 